\title{
Impact of 9/1 1-Related Chronic Conditions and PTSD Comorbidity on Early Retirement and Job Loss Among World Trade Center Disaster Rescue and Recovery Workers
}

\author{
Shengchao Yu, PhD, MA, ${ }^{1 *}$ Robert M. Brackbill, PhD, MPH, ${ }^{1}$ Sean Locke, MPH, ${ }^{1}$ \\ Steven D. Stellman, $\mathrm{PhD}^{\mathrm{MPH}}{ }^{2}$ and Lisa M. Gargano, $\mathrm{PhD}, \mathrm{MPH}^{1}$
}

Background The economic impact of the 9/11 terrorist attacks has rarely been studied. We examined the association between 9/11-related chronic health conditions with or without post-traumatic stress disorder (PTSD) and one important aspect of the economic impact, retirement, and job loss before age 60.

Methods A total of 7,662 workers who participated in the World Trade Center Health Registry surveys were studied. Logistic regression models examined the association of $9 /$ 11-related health and labor force exit.

Results Workers with chronic conditions were more likely to experience early retirement and job loss, and the association was stronger in the presence of PTSD comorbidity: the odds ratios for reporting early retirement or job loss were increased considerably when chronic conditions were comorbid with PTSD.

Conclusions Disaster-related health burden directly impacts premature labor force exit and income. Future evaluation of disaster outcome should include its long-term impact on labor force. Am. J. Ind. Med. 59:731-741, 2016. (c) 2016 Wiley Periodicals, Inc.

KEY WORDS: 9/11 health impact; post-traumatic stress disorder; comorbidity; early retirement; job loss; economic impact; disaster; employment

\section{INTRODUCTION}

The World Trade Center (WTC) September 11, 2001 attacks caused nearly 2,800 immediate deaths as well as short- and long-term health problems in those who survived. Severe respiratory effects such as asthma, and anxiety disorders including post-traumatic stress disorder (PTSD)

\footnotetext{
${ }^{1}$ New York City Department of Health and Mental Hygiene, Long Island City, New York ${ }^{2}$ Department of Epidemiology, Mailman School of Public Health, Columbia University, New York, New York

${ }^{*}$ Correspondence to: Shengchao Yu, PhD, MA, World Trade Center Health Registry, New York City Department of Health and Mental Hygiene, Gotham Center, 42-09 28th Street, 7th floor, Long Island City, NY 11101. E-mail: syu@ health.nyc.gov

Accepted 12 July 2016

DOI 10.1002/ajim.22640. Published online in Wiley Online Library (wileyonlinelibrary.com).
}

were reported by many investigators shortly after the attacks, while longer-term observations of a multitude of chronic physical and mental health conditions such as cardiovascular disease and cancer continue to be reported [Brackbill et al., 2009; Jordan et al., 2013; Boffetta et al., 2016].

The attacks also created economic distress for individuals, their families, and New York City (NYC) and the nation as a whole, but these consequences have been less well described. Studies of the economic impact of $9 / 11$ have largely been at the macro rather than individual level. It has been noted, for example, that aggregate NYC earnings losses of $\$ 2.8$ billion in the first 3 months [Dolfman and Wasser, 2004] and \$3.6-\$6.4 billion in the 9 months following the attacks were estimated for NYC industries [Bram et al., 2002], while macroeconomic analyses at the national level noted a general return of employment and consumer confidence in the short-term [Makinen, 2002; Roberts, 2009]. One study found 
that $47 \%$ of accidental disability retirements among NYC firefighters during the 7 years after 9/11 were for 9/11-related injuries or illnesses [Niles et al., 2011]. However, other than this study, little is known of the longer-term economic impact among the estimated 400,000 persons directly exposed to the collapse of the buildings and subsequent cleanup activities.

For individuals of working age, employment status is one key indicator of their socioeconomic standing and an important aspect of quality of life, and can be significantly compromised due to impaired functionality resulting from poor health. In a systematic review of the impact of poor health on labor force exit, van Rijn and colleagues found that self-perceived poor health was a risk factor for leaving paid employment through disability pension, unemployment, and early retirement [van Rijn et al., 2014]. Similar associations between self-perceived poor health and early retirement or unemployment were found in studies conducted in multiple European countries, Australia, and the United States as well [Dwyer and Mitchell, 1999; Mein et al., 2000; Karpansalo et al., 2004; Schuring et al., 2007; Alavinia and Burdorf, 2008; Pit et al., 2010; van den Berg et al., 2010; Rice et al., 2011; van Rijn et al., 2014]. Premature labor force exit has been associated with a variety of health conditions or symptoms. In the general population and in cancer survivors, suffering from depression, anxiety, or general mental health disorder has been associated with increased odds of early retirement, unemployment, or both [Haahr et al., 2007; Alavinia and Burdorf, 2008; Jokela et al., 2010; Pit et al., 2010; Rice et al., 2011; Olesen et al., 2012; Lindbohm et al., 2014; Singer et al., 2014]. Significant associations between respiratory, cardiovascular, musculoskeletal diseases, cancer and other chronic diseases, and early retirement were also found in different country settings [Haahr et al., 2007; Alavinia and Burdorf, 2008; Pit et al., 2010; Rice et al., 2011; Lindbohm et al., 2014; Singer et al., 2014].

In this study, we focused on retirement and job loss before age 60 for non-uniformed workers, one important aspect of the long-term economic impact of 9/11. We used longitudinal data collected by the WTC Health Registry. Rescue/recovery workers who reported that they were employed by the NYC Fire Department, Police Department, Department of Sanitation, or Department of Correction were excluded because uniformed workers from these Agencies are eligible for retirement benefits that allow them to retire with a pension based on $50 \%$ of final salary after certain years of service (required number of years in service ranges from 20 to 25 depending on the Agency rules) [Holloran and Castet, 2014; Nigro, 2015; NYCER, 2016]. In addition, uniformed workers are frequently eligible for accidental disability retirement through "World Trade Center Disability Law" enacted in 2006 by the New York State legislature (Chapter 445 of NYS Laws for 2006) with a pension based on $75 \%$ of final salary and is not federally taxable [Abbate, 2006], which was not available to other 9/11 rescue/recovery workers. Presumably, retirement pension options available to uniformed workers would influence their decision to retire early and differentiate them from the non-uniformed workers in our analysis.

Given the high prevalence of PTSD and comorbidity of PTSD with other chronic conditions among the 9/11 exposed population [Stellman et al., 2008; Brackbill et al., 2009; Chiu et al., 2011; Perlman et al., 2011; Nair et al., 2012; Friedman et al., 2013; Caramanica et al., 2014; Bromet et al., 2016], we hypothesized that early retirement and health-related job loss would be associated with 9/11-related chronic health problems, and that such associations would be greater in the presence of comorbid PTSD. The overall objective was to determine the long-term impact of 9/11 disaster-related health problems on labor force exit among exposed rescue and recovery workers. We further assessed the relationship between poor health and income drop among workers who experienced premature labor force exit.

\section{MATERIALS AND METHODS}

We studied relationships between 9/11 health problems experienced by rescue and recovery workers and two distinct modes of premature labor force exit, namely, early retirement and health-related job loss.

\section{Study Population}

The WTC Health Registry was established in 2002 to monitor the long-term health impact of the September 11, 2001 terrorist attack on the World Trade Center in New York City. In 2003-2004, the Registry conducted a health survey (Wave 1) with 71,431 enrollees consisting of rescue or recovery workers, Lower Manhattan residents, area workers, passers-by, and students or school staff. With the exception of enrollees who died or withdrew from the Registry, the entire adult cohort of the Registry was invited to participate in the Waves 2 and 3 surveys in 2006-2007 and again in 2011-2012. A more detailed description of the WTC Health Registry is provided elsewhere [Farfel et al., 2008; Brackbill et al., 2009]. The US Centers for Disease Control and Prevention and the New York City Department of Health and Mental Hygiene institutional review boards approved the Registry protocol, including use of the data.

This study focused on premature labor force exit as of Wave 3, and was limited to non-uniformed rescue and recovery workers consisting of volunteers, construction and utility company workers, staff from health care, finance, building management, and environmental services who participated in the WTC rescue, recovery, and clean-up operation, who had completed Waves 1,2 , and 3, who had not yet retired at Wave 2, and were under 60 years old at Wave 3 $(n=7,662)$. Since early retirement and health-related job loss 
are distinct pathways of premature labor force exit in this study, we excluded those who reported health-related job loss at Wave 3 from the early retirement analysis $(n=297)$, and similarly excluded those who reported early retirement at Wave 3 from the health-related job loss analysis $(n=291)$. In total, there were 50 workers who reported both early retirement and health-related job loss. The final study sample for early retirement analysis was 7,365, and the final sample for health-related job loss analysis was 7,112.

\section{Measures}

\section{Outcome measures: Early retirement and health-related job loss}

In both Waves 2 and 3, enrollees were asked about their current employment status including type of employment (such as full-time vs. part-time, self-employed, homemaker), retirement, and health-related unemployment, with no specific time given for the occurrence.

We focused on two labor force exit pathways, early retirement and health-related job loss, that could have occurred between Waves 2 and 3. First, we assessed the association between poor health and early retirement, defined as having retired before reaching age 60 as of Wave 3; and second, between poor health and health-related job loss, defined as being unable to work due to poor health before reaching age 60 as of Wave 3 . We restricted these analyses to enrollees who were employed at Wave 2 and who reported retirement or health-related job loss between Waves 2 and 3, in order to be reasonably certain that the health conditions preceded exit from the labor force and to exclude those marginally employable due to earlier health problems.

\section{Health measures at wave 2: Chronic health conditions and their comorbidity with PTSD}

At Wave 2, enrollees were asked whether they had ever been told by a doctor that they had any of more than a dozen listed health conditions. Chronic health conditions selected for this study included heart disease (angina, heart attack, or other heart disease), diabetes, depression, anxiety, lung disease (chronic bronchitis, emphysema, reactive airway disease, sarcoidosis, or other lung diseases), and asthma, all of which have been reported to be elevated among 9/11 exposed individuals [Stellman et al., 2008; Jordan et al., 2011a,b; Caramanica et al., 2014; Miller-Archie et al., 2014; Jordan et al., 2015]. Probable PTSD was also assessed at Wave 2 using a 9/11 specific PTSD Checklist-Civilian Version (PCL-17). As in prior published Registry analyses, enrollees with a cut-off score of 44 or greater were considered to have probable PTSD [Brackbill et al., 2009]. Comorbidity of chronic conditions and PTSD was defined as co-occurrence of any of these six types of self-reported diagnosed chronic conditions and probable PTSD.

The number of chronic health conditions, with and without PTSD, was included in the analytical models as the predictor of premature labor force exit.

\section{Socioeconomic characteristics and other covariates}

Socioeconomic characteristics, including sex, race, household income, education, and marital status collected from Wave 1 or 2, were included in the analysis for both early retirement and health-related job loss. Age was not assessed because we restricted the study sample to those who were younger than 60 years old, and the number of early retirements under the age of 40 was very low.

We also included a quantitative $9 / 11$ exposure measure that was previously found to be associated with PTSD in a dose-related manner [Brackbill et al., 2009]. This measure, which has been described in detail [Caramanica et al., 2014], is a 12-item summary measure that combines information from both Waves 1 and 2, including exposure to dust cloud, injury, witnessing horror, bereavement, and home evacuation. A score of zero or one was categorized as low/no exposure, $2-3$ as medium, $4-5$ as high, and six or greater as very high.

\section{Statistical Analyses}

Separate logistic regression models were used to examine associations between 9/11-related poor health and the two premature labor force exit outcomes: early retirement and health-related job loss. Socioeconomic characteristics and other covariates were controlled in the logistic regression models. The correlation of selected chronic conditions and self-assessed health was tested but not shown in the Results.

Income changes since 9/11 and how the changes were associated with health status were estimated for rescue and recovery workers who had retired early or who had healthrelated job loss during Waves 2 and 3. We hypothesize that 9/11-related chronic conditions and their comorbidity with PTSD may have been associated with both higher likelihood of income drop and increased involuntary labor force exit for rescue and recovery workers. Analyses were conducted in SAS version 9.4 (SAS Institute Inc., Cary, NC).

\section{RESULTS}

\section{Early Retirement, Chronic Health Conditions, and PTSD Comorbidity}

Among 7,365 rescue and recovery workers who had not retired at Wave 2, $241(3.3 \%)$ reported having retired 
between Waves 2 and 3 before they reached the age of 60 (Table I).

Four sociodemographic characteristics of the rescue and recovery workers were significantly associated with early retirement: race, 2002 household income, education, and marital status. Non-Hispanic black workers showed higher odds of reporting early retirement than non-Hispanic white workers. The odds of reporting early retirement increased significantly by 2.7 (95\%CI: 1.2-6.2), 4.5 (95\%CI: $2.0-10.0$ ), and 5.6 (95\% CI: $2.3-13.8)$ times for individuals from the three high income groups $(\$ 50,000-\$ 75,000, \$ 75,000-\$ 150,000$, and above $\$ 150,000$ ) as compared to those with household income lower than $\$ 25,000$. On the contrary, the odds of retiring before reaching the age of 60 dropped by $40 \%$ $(\mathrm{AOR}=0.6,95 \% \mathrm{CI}: 0.4-0.8)$ and $70 \%(\mathrm{AOR}=0.3,95 \% \mathrm{CI}$ : $0.2-0.6$ ), respectively, for those with a college or postgraduate degree compared to those with high school and below education. Being divorced or separated increased the odds of early retirement by 1.7 (95\%CI: $1.2-2.6)$ relative to those staying married or living with a partner.

As shown in Table I, having two of the six chronic conditions without PTSD was significantly associated with higher odds of experiencing early retirement relative to those with no chronic conditions or PTSD (AOR $=1.9,95 \%$ CI: 1.2-2.9). The odds of early retirement did not increase significantly for those with three or more conditions without PTSD, those with PTSD alone, or those with PTSD comorbid with one other condition, probably because of small numbers of cases in these categories.

PTSD comorbid with two or more other chronic conditions was associated with greater likelihood of early retirement. For example, the odds of reporting early retirement for those with two conditions without PTSD, as compared to workers with none of the selected conditions at all, was 1.9 (95\%CI: 1.2-2.9) and was increased to 2.4 with addition of PTSD (95\%CI: 1.3-4.2). Similarly, for those with three or more conditions, the odds of early retirement grew from 1.3 (not significant) to 2.1 (95\%CI: 1.2-3.9) with addition of PTSD, as compared to those without any of the selected conditions.

\section{Health-Related Job Loss, Chronic Health Conditions, and PTSD Comorbidity}

Of the 7,112 rescue and recovery workers with complete job loss data, 247 (3.5\%) reported health-related job loss at Wave 3 (Table II).

In contrast to early retirement, there was an inverse association between health-related job loss and household income. More specifically, when household income exceeded $\$ 75,000$, the odds of health-related job loss were reduced by more than half as compared to those having household income below $\$ 25,000$ ( $\mathrm{AOR}=0.4,95 \%$
CI: $0.2-0.6)$, suggesting that the financially disadvantaged groups were more likely to experience health-related job loss. Those with a post-graduate education were less likely to experience health-related job loss than those with high school education or below (AOR $=0.5,95 \% \mathrm{CI}$ : $0.3-0.8$ ). In addition, the odds of health-related job loss for workers with very high exposure during 9/11 doubled as compared to those with no or low exposure, even after controlling for other variables including health conditions $(\mathrm{AOR}=2.0$, 95\%CI: 1.2-3.2).

Among those with three or more chronic illnesses, the odds of health-related job loss were three times higher than those with no selected health condition (AOR $=3.2,95 \% \mathrm{CI}$ : 1.6-6.5). When chronic illness was comorbid with PTSD, the odds of experiencing job loss was greater. For example, workers with PTSD comorbid with three or more other conditions were more than 10 times more likely to experience health-related job loss than those with none of the selected health conditions (AOR $=10.7,95 \% \mathrm{CI}$ : 6.7-17.2). In addition, even PTSD alone (with no other major comorbid condition) could almost triple workers' likelihood of having health-related job loss $(\mathrm{AOR}=2.6$, 95\%CI: 1.4-5.1).

\section{Health Conditions and Income Drop Post-Labor Force Exit}

Household income was asked in both Waves 1 and 3, and was measured in five categories, from below $\$ 25,000$, $\$ 25,000-\$ 50,000, \$ 50,000-\$ 75,000, \$ 75,000-\$ 150,000$, to above $\$ 150,000$. Income drop in this analysis was defined as a change in income from a higher category in 2002 to a lower category in 2010 . On average, $18.6 \%$ of Registry enrollees experienced one or more categories of income drop from 2002 to 2010. Among rescue and recovery workers who retired early between Waves 2 and 3, the percentage was higher, ranged from $35.1 \%$ to $51.0 \%$, depending on their health status at Wave 2 (Fig. 1). For those without PTSD and did not have any of the six major chronic conditions and those who had at least one of these conditions, the percentages with income category drop were more similar (35.1\% vs. $39.8 \%)$. However, for early retirees who had PTSD in addition to at least one other condition, the percentage with income category drop increased remarkably to $51.0 \%$.

Among workers who experienced health-related job loss between Waves 2 and 3, having at least one of the six major chronic conditions but without PTSD was associated with higher percentage of income category drop (43.8\%), as compared to those without any major health condition (25.6\%). Furthermore, among those with PTSD comorbid with other conditions, more than half of them experienced income category drop (51.6\%). 
TABLE I. Early Retirement, Chronic Conditions and PTSD Comorbidity, and Other Characteristics Among 7,365 Rescue and Recovery Workers

\begin{tabular}{|c|c|c|c|c|c|c|}
\hline & \multicolumn{4}{|c|}{ Retired early } & & \\
\hline & \multicolumn{2}{|c|}{ No (N, \%) } & \multicolumn{2}{|c|}{ Yes (N, \%) } & \multicolumn{2}{|c|}{ Likelihood of early retirement } \\
\hline & 7,124 & 96.7 & 241 & $\overline{3.3}$ & $\mathrm{AOR}^{\mathrm{a}}$ & $95 \% \mathrm{CI}^{\mathrm{b}}$ \\
\hline \multicolumn{7}{|l|}{ Sex } \\
\hline Female & 2,097 & 97.5 & 53 & 2.5 & & \\
\hline Male & 5,027 & 96.4 & 188 & 3.6 & 1.2 & $0.8,1.7$ \\
\hline \multicolumn{7}{|l|}{ Race } \\
\hline Non-Hispanic white & 5,336 & 96.8 & 179 & 3.2 & Reference & \\
\hline Non-Hispanic black & 461 & 93.9 & 30 & 6.1 & 2.2 & $1.4,3.4^{* *}$ \\
\hline Hispanic & 862 & 97.4 & 23 & 2.6 & 1.0 & $0.6,1.6$ \\
\hline Asian & 229 & 98.3 & 4 & 1.7 & 0.5 & $0.2,1.7$ \\
\hline Other-multi-racial & 236 & 97.9 & 5 & 2.1 & 1.0 & $0.4,2.4$ \\
\hline \multicolumn{7}{|l|}{ Total household Income in 2002, \$ } \\
\hline$<25,000$ & 581 & 98.8 & 7 & 1.2 & Reference & \\
\hline $25,000 \leq 50,000$ & 1,365 & 98.6 & 19 & 1.4 & 1.0 & $0.4,2.5$ \\
\hline $50,000 \leq 75,000$ & 1,581 & 96.8 & 52 & 3.2 & 2.7 & $1.2,6.2^{*}$ \\
\hline $75,000 \leq 150,000$ & 2,563 & 95.5 & 120 & 4.5 & 4.5 & $2.0,10.0^{* *}$ \\
\hline$\geqq 150,000$ & 547 & 95.8 & 24 & 4.2 & 5.6 & $2.3,13.8^{* *}$ \\
\hline \multicolumn{7}{|l|}{ Education } \\
\hline High school and below & 1,699 & 95.6 & 79 & 4.4 & Reference & \\
\hline College & 3,986 & 96.8 & 130 & 3.2 & 0.6 & $0.4,0.8^{* *}$ \\
\hline Post-graduate & 1,404 & 97.8 & 31 & 2.2 & 0.3 & $0.2,0.6^{* * *}$ \\
\hline \multicolumn{7}{|l|}{ Marital status } \\
\hline Married or living with partner & 5,059 & 96.6 & 176 & 3.4 & Reference & \\
\hline Divorced or separated & 752 & 95.1 & 39 & 4.9 & 1.7 & $1.2,2.6^{* *}$ \\
\hline Widowed & 42 & 93.3 & 3 & 6.7 & 2.2 & $0.5,9.7$ \\
\hline Never married & 1,222 & 98.3 & 21 & 1.7 & 0.7 & $0.4,1.2$ \\
\hline \multicolumn{7}{|l|}{ Disaster exposure } \\
\hline Low/none & 2,243 & 96.7 & 76 & 3.3 & & \\
\hline Medium & 2,958 & 96.6 & 103 & 3.4 & 0.8 & $0.6,1.1$ \\
\hline High & 1,322 & 96.7 & 45 & 3.3 & 0.8 & $0.5,1.2$ \\
\hline Very high & 601 & 97.2 & 17 & 2.8 & 0.7 & $0.4,1.3$ \\
\hline \multicolumn{7}{|c|}{ Number of chronic conditions ${ }^{c}$ without PTSD } \\
\hline 0 & 3,187 & 97.3 & 88 & 2.7 & Reference & \\
\hline 1 & 1,447 & 96.7 & 50 & 3.3 & 1.3 & $0.9,1.9$ \\
\hline 2 & 717 & 95.9 & 31 & 4.1 & 1.9 & $1.2,2.9^{* *}$ \\
\hline$\geqq 3$ & 293 & 96.7 & 10 & 3.3 & 1.3 & $0.6,2.7$ \\
\hline \multicolumn{7}{|c|}{ Number of chronic conditions ${ }^{\mathrm{C}}$ with PTSD } \\
\hline 0 & 323 & 97.0 & 10 & 3.0 & 1.3 & $0.6,2.5$ \\
\hline 1 & 300 & 95.8 & 13 & 4.2 & 1.6 & $0.9,3.0$ \\
\hline 2 & 317 & 95.2 & 16 & 4.8 & 2.4 & $1.3,4.2^{* *}$ \\
\hline$\geqq 3$ & 353 & 95.4 & 17 & 4.6 & 2.1 & $1.2,3.9^{*}$ \\
\hline
\end{tabular}

${ }^{*} P<0.05 ; * * P<0.01 ; * * * P<0.0001$.

${ }^{a} A 0 R$ : adjusted odds ratio and was adjusted for all factors listed in this table.

${ }^{\mathrm{b}} 95 \% \mathrm{Cl}$ : 95\% confidence interval.

${ }^{\circ}$ Chronic health conditions include heart diseases, diabetes, depression, anxiety, lung diseases, and asthma. 
TABLE II. Health-Related Job Loss, Chronic Conditions and PTSD Comorbidity, and Other Characteristics Among 7,112 Rescue and Recovery Workers

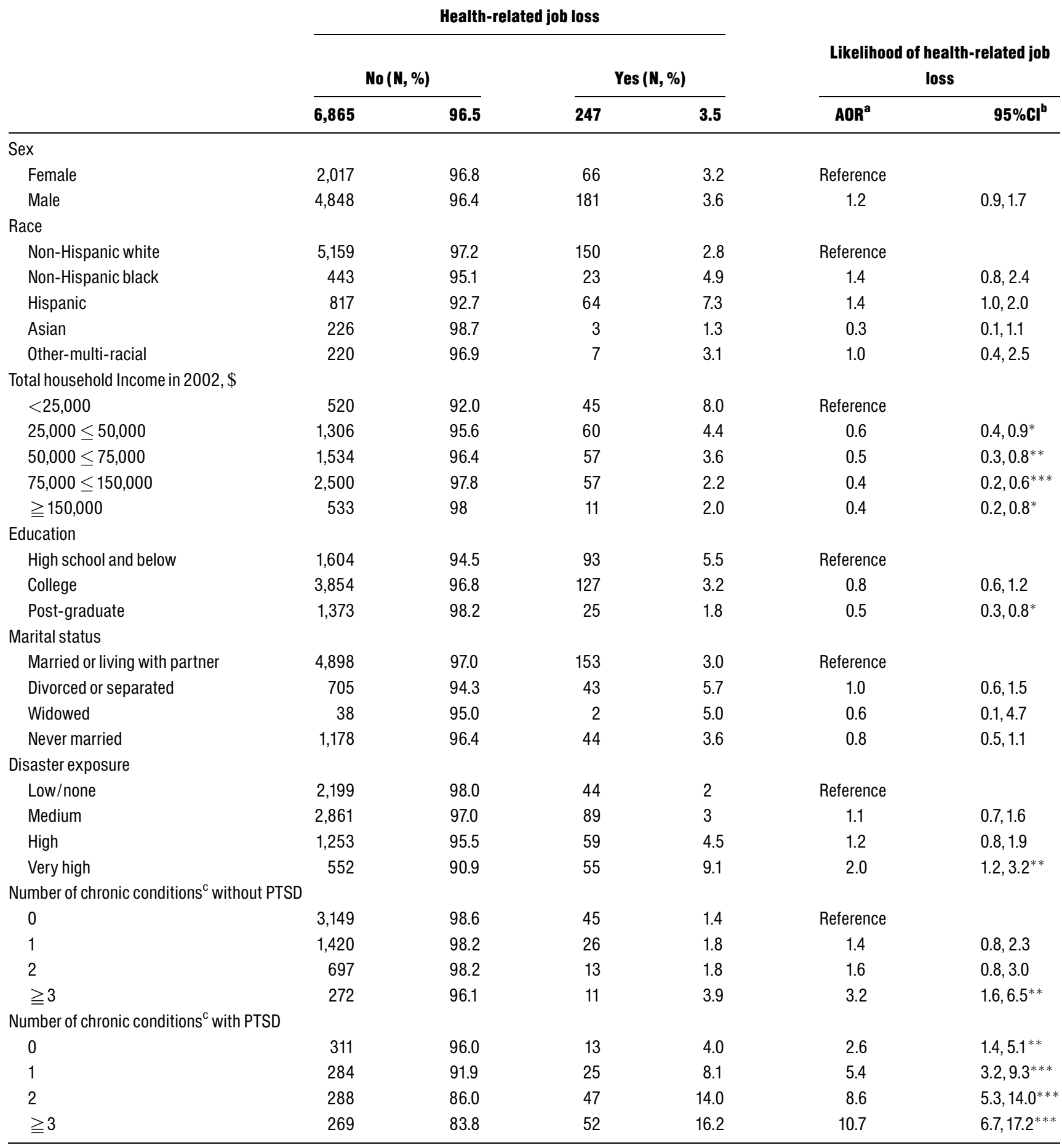

${ }^{*} P<0.05$; $^{* *} P<0.01 ;^{* * *} P<0.0001$.

${ }^{\mathrm{a} A O R}$ : adjusted odds ratio and was adjusted for all factors listed in this table.

${ }^{b} 95 \% \mathrm{Cl}$ : 95\% confidence interval.

${ }^{c}$ Chronic health conditions include heart diseases, diabetes, depression, anxiety, lung diseases, and asthma. 


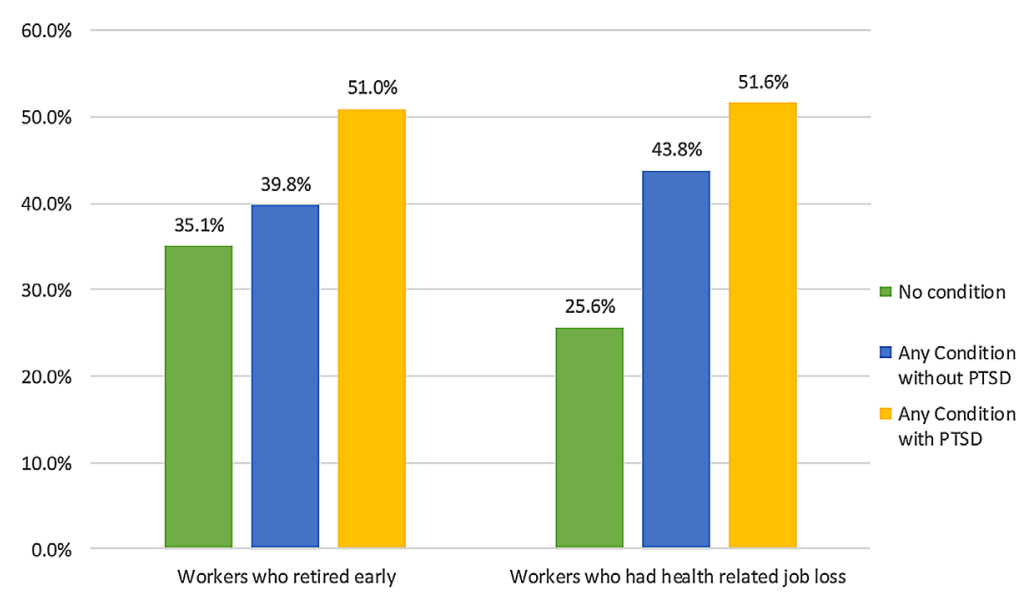

FIGURE 1. Percentage of those with income drop between years 2002 and 2010 by chronic health condition* (*including heart disease, diabetes, depression, anxiety, lung disease, and asthma): among two rescue and recovery worker subgroups.

\section{DISCUSSION}

In contrast to the focus on its health and environmental outcomes, the impact of the 9/11 disaster on premature labor force exit has rarely been studied until now. This paper examined employment adjustment up to 10 years after the disaster for non-uniformed rescue and recovery workers. We found that both early retirement and health-related job loss that occurred between 5 and 10 years after the disaster were positively associated with 9/11-related chronic health conditions, and the association was even stronger when these chronic conditions were comorbid with probable PTSD.

Between 2006/2007 and 2011/2012, that is between 5 and 10 years after the disaster, about $7 \%$ of the nonuniformed rescue and recovery workers in the WTC Health Registry left employment prematurely $(3.3 \%$ via early retirement and $3.5 \%$ via health-related job loss, Tables I and II). Workers who endured the most serious 9/11-related health burden were those who retired early before reaching the age of 60, and were also most likely to be unemployed because of health reasons. Furthermore, when their health conditions were comorbid with 9/11-related probable PTSD, the chances of these workers experiencing early retirement as much as doubled, and the odds of healthrelated job loss increased as much as over tenfold, as compared to the workers with no major chronic condition or probable PTSD.

Large scale disasters often cause traumatic stress on people exposed [Caramanica et al., 2015]. In the case of the 9/11 attack in NYC, numerous studies have shown that probable PTSD has persisted many years after the disaster [Brackbill et al., 2009; Pietrzak et al., 2014; Cone et al., 2015; Maslow et al., 2015], and a number of 9/11-related chronic conditions or substance abuse were all strongly correlated with PTSD or co-existed with PTSD [Perlman et al., 2011;
Nair et al., 2012; Friedman et al., 2013; Jordan et al., 2013; Caramanica et al., 2014; Welch et al., 2015]. Studies that have assessed the association between poor health and premature labor force exit have rarely noted the influence of comorbid mental and physical health conditions on employment status [Dwyer and Mitchell, 1999; Mein et al., 2000; Karpansalo et al., 2004; Schuring et al., 2007; Alavinia and Burdorf, 2008; Jokela et al., 2010; Pit et al., 2010; van den Berg et al., 2010; Rice et al., 2011; Olesen et al., 2012; Lindbohm et al., 2014; van Rijn et al., 2014]. Of these the only study that found comorbid mental health as a risk factor for early retirement was a study of a small number of cancer patients from four hospitals in Germany [Singer et al., 2014]. In assessing long-term impact of disaster on employment adjustment, our study demonstrated the significant effect of mental health, and emphasized the need of involving disaster triggered mental and physical health comorbidity in future analyses.

We hypothesize based on our results that there are two pathways to exiting labor force for rescue and recovery workers, early retirement and health-related job loss. The decision to retire or retire early is complicated and often influenced by not only health status but also leisure interests [Shultz et al., 1998; Ferrie et al., 2014]. Some may voluntarily retire early for leisure where good health and/ or adequate finance could lead to early retirement [Shultz et al., 1998; De Wind et al., 2013; Robroek et al., 2013]. Others, such as rescue and recovery workers in our study might have retired, voluntarily or involuntarily, due to ill health, where high income or adequate financial resources would have allowed them to retire early anyway. This hypothesis was supported by the positive relationship between poor health and early retirement, and between income and early retirement in our findings, and is consistent with other earlier studies [Mein et al., 2000; Singer et al., 2014]. On the other hand, our study showed that 
some workers would try to stay in labor force until eventually being forced out due to deteriorating health. These workers eventually left their jobs involuntarily because their low income or education levels and poor health were not successful in maintaining work. This finding was also in line with other research on the socioeconomic inequalities of health, which suggested individuals with lower socioeconomic status were usually at higher risk of involuntary labor force exit [McDonough and Amick, 2001; Robroek et al., 2015].

Our study further examined the interwoven relationship of income loss and poor health for those who underwent premature labor force exit. Although it is not surprising to observe income loss after leaving the labor force, our results illustrated that, the least healthy individuals were most likely to experience income drop over years. This was true for both early retirement and job loss especially among those who suffered comorbidity of PTSD and other chronic conditions, despite the fact that people selected into early retirement were those with relatively more financial resources than those leaving job due to health. We conjecture that the relatively healthier workers may be more likely to offset income loss by taking income generating work after leaving the labor force, as compared to those suffering comorbidity of PTSD and other chronic conditions. High health care expenditures are also expected to reduce net worth of those with comorbidity more than for those with no disease [Kim et al., 2015], although we do not have data on net wealth to explore in this aspect. Our findings suggest that once workers with comorbidity of PTSD and other chronic conditions exited the labor force, their long-term and overall well-being may have been continuously compromised through the co-occurrence of poor health and gradual income reduction, even though some of the early retirees might have enjoyed relatively high income before.

The two potential pathways of premature labor force exit identified in our study, early retirement and healthrelated job loss, do not necessarily apply to uniformed rescue and recovery workers (majority of whom were first responders of the disaster, e.g., those from FDNY or NYPD), a group not included in this analysis. Although uniformed workers experienced documented 9/11-related ill health, they have retirement pension options unavailable to non-uniformed workers that likely influences their decision on whether or not to retire and when to retire. It is also unlikely that uniformed workers would leave the labor force by job loss. Also, the financial impact of retirement for uniform workers is frequently offset by generous pension packages, as well as retirement at a relatively young age, giving them the opportunity for income generating work post-retirement. Thus, the complicated nature of retirement and the potential financial impact for uniformed workers are beyond the scope of this analysis and are worthy of a separate in-depth study.
This study focused on how disaster-related poor health was associated with subsequent labor force exit, and how socioeconomic status played a role in the process. Although accidental disability retirements have been attributed to WTC-related illnesses and injuries among FDNY firefighters [Niles et al., 2011], early retirement and job loss observed in this study cannot be attributed solely to $9 / 11$ exposures or subsequent 9/11-related impaired health due to lack of a comparison group of rescue and recovery workers who have job-related occupational risks independent of 9/11 exposures. Future studies may address this limitation by collecting more information on occupational exposures and other non-9/11-related health risk factors. A further limitation is that we did not have information on the exact dates of retirement or job loss; the temporal relationship between poor health and labor force outcomes was established by using the health data collected in 2006-2007 from the Wave 2 survey, and by limiting labor force exits to those occurred after the Wave 2 survey data collection and before the Wave 3 survey, that is, between 2006/2007 and 2011/2012. Use of broad categories of reported income limited assessment of income drop to fairly large changes between categories, overlooking smaller income losses within the same category. Therefore, the relationship between income drop and health condition reported in this study applies to those with fairly substantial income loss during 2002 and 2010, and is likely to underestimate the extent of income loss in the overall population. Overtime compensation associated with work at the WTC sites in the year after 9/11 in addition to overtime compensation in the years before retirement can also reduce any measure of income drop. Our analyses could not control for this factor due to lack of data, but we think overtime would be a more serious confounding element for uniformed workers than non-uniformed workers.

Among over 30,000 rescue and recovery workers in the WTC Health Registry cohort, 90\% were still under the conventional retirement age of 65 as of 2012, about 10 years after the disaster. Assessment of employment status change over time for such a large group should become an important component of further research on the overall impact of the 9/11 disaster. Furthermore, this analysis focused on nonuniformed rescue and recovery workers, a vulnerable but generally understudied group mainly consisting of volunteers, construction and utility company workers, staff from health care, finance, building management, and environmental services, who were not regularly trained for emergency service, and who were not eligible for variety of retirement options at a relatively young age. As we found here, disasters tend to have a large ripple effect on their overall well-being through health, employment, and earnings. Future evaluation of disaster impact should extend beyond the direct and shortterm health consequences and include its long-term impact on labor force as well. 


\section{AUTHORS' CONTRIBUTIONS}

All authors have read, contributed to, and approved this manuscript. Shengchao Yu, Robert Brackbill, and Steven Stellman conceived the study. Shengchao Yu performed the coding and statistical analysis of the data with input from Sean Locke. Shengchao Yu wrote the manuscript with editing input from Steven Stellman, Lisa Gargano, Robert Brackbill, and Sean Locke. All authors discussed the results and implications and commented on the manuscript at all stages.

\section{ACKNOWLEDGMENTS}

The authors thank Dr. Cheryl Stein for her thorough proof on the SAS codes for this paper, and Drs. Mark Farfel, Sharon Perlman, Charon Gwynn, James Hadler, and anonymous reviewers for their critical review of this paper, and Dr. Deborah Walker, David Wu, Lennon Turner, and other Registry staff who helped maintain the World Trade Center Health Registry cohort.

\section{FUNDING}

This work was supported by Cooperative Agreement (Number 5U50/OH009739) from the Centers for Disease Control and Prevention (CDC) - the National Institute for Occupational Safety and Health (NIOSH); and from the Agency for Toxic Substances and Disease Registry (ATSDR) (U50/ATU272750) of the CDC, which included support from CDC - the National Center for Environmental Health (NCEH) and the New York City Department of Health and Mental Hygiene (NYC DOHMH). Its contents are solely the responsibility of the authors and do not necessarily represent the official views of NIOSH-CDC.

\section{ETHICS APPROVAL AND INFORMED CONSENT}

The analytical data of this paper is from the World Trade Center Health Registry (WTCHR) Surveys. All participants in the Surveys gave verbal informed consent to participate in the WTCHR. The US Centers for Disease Control and Prevention and the New York City Department of Health and Mental Hygiene institutional review boards approved the WTCHR protocol, including use of the data.

\section{DISCLOSURE (AUTHORS)}

The authors declare no conflict of interest.

\section{DISCLOSURE BY AJIM EDITORS OF RECORD}

Paul Landsbergis declares that he has no competing or conflicts of interest in the review and publication decision regarding this article.

\section{DISCLAIMER}

None.

\section{REFERENCES}

Abbate PJJ. 2006. 2005-2006 Annual Report of the Committee on Governmental Employees: New York State Assembly.

Alavinia SM, Burdorf A. 2008. Unemployment and retirement and illhealth: A cross-sectional analysis across European countries. Int Arch Occup Environ Health 82(1):39-45.

Boffetta P, Zeig-Owens R, Wallenstein S, Li J, Brackbill R, Cone J, Farfel M, Holden W, Lucchini R, Webber MP, et al. 2016. Cancer in World Trade Center responders: Findings from multiple cohorts and options for future study. Am J Ind Med 59(2):96-105.

Brackbill RM, Hadler JL, DiGrande L, Ekenga CC, Farfel MR, Friedman S, Perlman SE, Stellman SD, Walker DJ, Wu D, et al. 2009. Asthma and posttraumatic stress symptoms 5 to 6 years following exposure to the World Trade Center terrorist attack. JAMA 302(5): $502-516$.

Bram J, Orr J, Rapaport C. 2002. Measuring the effects of the September 11 attack on New York City. Econ Policy Rev 8(2):5-20.

Bromet E, Hobbs M, Clouston S, Gonzalez A, Kotov R, Luft B. 2016. Dsm-Iv post-traumatic stress disorder among World Trade Center responders 11-13 years after the disaster of 11 September $2001(9 / 11)$. Psychol Med 46(04):771-783.

Caramanica K, Brackbill RM, Liao T, Stellman SD. 2014. Comorbidity of 9/11-related ptsd and depression in the World Trade Center Health Registry10-11. years postdisaster. J Trauma Stress 27(6):680-688.

Caramanica K, Brackbill RM, Stellman SD, Farfel MR. 2015. Posttraumatic stress disorder after hurricane Sandy among persons exposed to the 9/11 disaster. Int J Emerg Ment Health 17(1):356.

Chiu S, Niles JK, Webber MP, Zeig-Owens R, Gustave J, Lee R, Rizzotto L, Kelly KJ, Cohen HW, Prezant DJ. 2011. Evaluating risk factors and possible mediation effects in posttraumatic depression and posttraumatic stress disorder comorbidity. Public Health Rep 126: 201-209.

Cone JE, Li J, Kornblith E, Gocheva V, Stellman SD, Shaikh A, Schwarzer R, Bowler RM. 2015. Chronic probable ptsd in police responders in the World Trade Center Health Registry ten to eleven years after 9/11. Am J Ind Med 58(5):483-493.

De Wind A, Geuskens GA, Reeuwijk KG, Westerman MJ, Ybema JF, Burdorf A, Bongers PM, Van der Beek AJ. 2013. Pathways through which health influences early retirement: A qualitative study. BMC Public Health 13(1):292.

Dolfman ML, Wasser SF. 2004. 9/11 and the New York City economy: A borough-by-borough analysis. Mon Labor Rev 127(3):3-33.

Dwyer DS, Mitchell OS. 1999. Health problems as determinants of retirement: Are self-rated measures endogenous? J Health Econ 18(2):173-193. 
Farfel M, DiGrande L, Brackbill R, Prann A, Cone J, Friedman S, Walke DJ, Pezeshki G, Thomas P, Galea S, et al. 2008. An overview of 9/11 experiences and respiratory and mental health conditions among World Trade Center Health Registry enrollees. J Urban Health 85(6):880-909.

Ferrie JE, Virtanen M, Kivimaki M. 2014. The healthy population-high disability paradox. Occup Environ Med 71(4):232-233.

Friedman SM, Farfel MR, Maslow CB, Cone JE, Brackbill RM, Stellman SD. 2013. Comorbid persistent lower respiratory symptoms and posttraumatic stress disorder 5-6 years post-9/11 in responders enrolled in the World Trade Center Health Registry. Am J Ind Med 56(11):1251-1261.

Haahr JPL, Frost P, Andersen JH. 2007. Predictors of health-related job loss: A two-Year follow-up study in a general working population. J Occup Rehabil 17(4):581-592.

Holloran K, Castet R-S. 2014. Summary Plan Description New York City Police Pension Fund Tier 3 Members the New York City Police Pension Fund. p. 14.

Jokela M, Ferrie JE, Gimeno D, Chandola T, Shipley MJ, Head J, Vahtera J, Westerlund H, Marmot MG, Kivimäki M. 2010. From midlife to early old age: Health trajectories associated with retirement. Epidemiology (Cambridge, Mass) 21(3):284-290

Jordan HT, Miller-Archie SA, Cone JE, Morabia A, Stellman SD 2011a. Heart disease among adults exposed to the september 11, 2001 World Trade Center disaster: Results from the World Trade Center Health Registry. Prev Med 53(6):370-376.

Jordan HT, Stellman SD, Prezant D, Teirstein A, Osahan SS, Cone JE. 2011b. Sarcoidosis diagnosed after september 11, 2001, among adults exposed to the World Trade Center disaster. J Occup Environ Med 53(9):966-974.

Jordan HT, Stellman SD, Morabia A, Miller-Archie SA, Alper H, Laskaris Z, Brackbill RM, Cone JE. 2013. Cardiovascular disease hospitalizations in relation to exposure to the september 11, 2001 world trade center disaster and posttraumatic stress disorder. J Am Heart Assoc 2(5):e000431.

Jordan HT, Stellman SD, Reibman J, Farfel MR, Brackbill RM, Friedman SM, Li J, Cone JE. 2015. Factors associated with poor control of 9/11-related asthma 10-11 years after the 2001 world trade center terrorist attacks. J Asthma 52(6):1-8.

Karpansalo M, Manninen P, Kauhanen J, Lakka TA, Salonen JT. 2004 Perceived health as a predictor of early retirement. Scand J Work Environ Health 30(4):287-292.

Kim H, Shin S, Zurlo KA. 2015. Sequential patterns of health conditions and financial outcomes in late life: Evidence from the health and retirement study. Int J Aging Hum Dev 81(1-2):54-82.

Lindbohm M-L, Kuosma E, Taskila T, Hietanen P, Carlsen K Gudbergsson S, Gunnarsdottir H. 2014. Early retirement and nonemployment after breast cancer. Psychooncology 23(6):634-641.

Makinen G. 2002. The Economic Effects of 9/11: A Retrospective Assessment. Library of Congress Washington DC Congressional Research Service.

Maslow CB, Caramanica K, Welch AE, Stellman SD, Brackbill RM, Farfel MR. 2015. Trajectories of scores on a screening instrument for ptsd among World Trade Center rescue, recovery, and clean-up workers. J Trauma Stress 28(3):198-205.

McDonough P, Amick BC. 2001. The social context of health selection: A longitudinal study of health and employment. Soc Sci Med 53(1):135-145.

Mein G, Martikainen P, Stansfeld SA, Brunner EJ, Fuhrer R, Marmot MG. 2000. Predictors of early retirement in british civil servants. Age Ageing 29(6):529-536.
Miller-Archie SA, Jordan HT, Ruff RR, Chamany S, Cone JE, Brackbill RM, Kong J, Ortega F, Stellman SD. 2014. Posttraumatic stress disorder and new-onset diabetes among adult survivors of the world trade center disaster. Prev Med 66:34-38.

Nair HP, Ekenga CC, Cone JE, Brackbill RM, Farfel MR, Stellman SD 2012. Co-occurring lower respiratory symptoms and posttraumatic stress disorder 5 to 6 years after the World Trade Center terrorist attack. Am J Public Health 102(10):1964-1973.

Nigro DA. 2015. Comprehensive Annual Financial Report: A Pension Trust Fund of the City of New York for Fiscal Years Ended June 30, 2015 and June 30, 2014. New York Fire Department Pension Funds. p. 49.

Niles J, Webber M, Gustave J, Zeig-Owens R, Lee R, Glass L, Weiden MD, Kelly K, Prezant DJ. 2011. The impact of the World Trade Center attack on fdny firefighter retirement, disabilities, and pension benefits. Am J Ind Med 54(9):672-680.

NYCER 2016. 22-Year Plan Fact Sheet: New York City employees' retirement system.

Olesen SC, Butterworth P, Rodgers B. 2012. Is poor mental health a risk factor for retirement? findings from a longitudinal population survey. Soc Psychiatry Psychiatr Epidemiol 47(5):735-744.

Perlman SE, Friedman S, Galea S, Nair HP, Erős-Sarnyai M, Stellman SD, Hon J, Greene CM. 2011. Short-term and medium-term health effects of 9/11. Lancet 378(9794):925-934.

Pietrzak R, Feder A, Singh R, Schechter CB, Bromet EJ, Katz C, Reissman D, Ozbay F, Sharma V, Crane M. 2014. Trajectories of ptsd risk and resilience in World Trade Center responders: An 8-year prospective cohort study. Psychol Med 44(01):205-219.

Pit SW, Shrestha R, Schofield D, Passey M. 2010. Health problems and retirement due to ill-health among australian retirees aged 45-64 years. Health Policy 94(2):175-181.

Rice NE, Lang IA, Henley W, Melzer D. 2011. Common health predictors of early retirement: Findings from the english longitudinal study of ageing. Age Ageing 40(1):54-61.

Roberts BW. 2009. The macroeconomic impacts of the 9/11 attack: Evidence from real-time forecasting. Peace Econ Peace Sci Pub Pol 15(2):ISSN (Online) 1554-8597.

Robroek SJ, Schuring M, Croezen S, Stattin M, Burdorf A. 2013. Poor health, unhealthy behaviors, and unfavorable work characteristics influence pathways of exit from paid employment among older workers in europe: A four year follow-up study. Scand J Work Environ Health 39(2):125-133.

Robroek SJ, Rongen A, Arts CH, Otten FW, Burdorf A, Schuring M. 2015. Educational inequalities in exit from paid employment among dutch workers: The influence of health, lifestyle and work. PLoS ONE 10(8):e0134867.

Schuring M, Burdorf L, Kunst A, Mackenbach J. 2007. The effects of ill health on entering and maintaining paid employment: Evidence in European countries. J Epidemiol Community Health 61(7):597-604.

Shultz KS, Morton KR, Weckerle JR. 1998. The influence of push and pull factors on voluntary and involuntary early retirees' retirement decision and adjustment. J Vocat Behav 53(1):45-57.

Singer S, Meyer A, Wienholz S, Briest S, Brown A, Dietz A, Binder H, Jonas S, Papsdorf K, Stolzenburg JU. 2014. Early retirement in cancer patients with or without comorbid mental health conditions: A prospective cohort study. Cancer 120(14):2199-2206.

Stellman JM, Smith RP, Katz CL, Sharma V, Charney DS, Herbert R, Moline J, Luft BJ, Markowitz S, Udasin I. 2008. Enduring mental health morbidity and social function impairment in World Trade Center rescue, recovery, and cleanup workers: The psychological dimension of an environmental health disaster. Environ Health Perspect 116(9): $1248-1253$. 
Welch AE, Jasek JP, Caramanica K, Chiles MC, Johns M. 2015. Cigarette smoking and 9/11-related posttraumatic stress disorder among World Trade Center Health Registry enrollees, 2003-2012. Prevent Med 73:94-99.

van Rijn RM, Robroek SJ, Brouwer S, Burdorf A. 2014. Influence of poor health on exit from paid employment: A systematic review. Occup Environ Med 71(4):295-301. van den Berg T, Schuring M, Avendano M, Mackenbach J, Burdorf A. 2010. The impact of ill health on exit from paid employment in europe among older workers. Occup Environ Med 67(12):845-852.

Institution at which the work was performed: New York City Department of Health and Mental Hygiene. 